\title{
HyperCard administration of a block-design task
}

\author{
THOMAS A. MARTIN and KATHRYN L. WILCOX \\ Susquehanna University, Selinsgrove, Pennsylvania
}

\begin{abstract}
HyperCard (Atkinson, 1987) is a new development environment for the Macintosh that shows promise for use in psychological research and testing. In this paper, we discuss the development of HyperCard stackware with which a block-design task similar to that of the Wechsler Adult Intelligence Scale-Revised (WAIS-R) can be administered. The reliability and validity of the computerized block-design task was evaluated by administering both the computerized task and the WAIS-R subtest to college undergraduates. Results indicated that the computerized task's reliability compared favorably with that of the WAIS-R subtest. Validity coefficients were equivocal; although an elapsed-time measure showed moderate correlation between the tasks, the numbers of designs correctly completed in each condition were not significantly correlated.
\end{abstract}

The release of HyperCard for the Apple Macintosh was accompanied by enthusiastic media coverage, in both trade magazines (Goodman, 1987; Saffo, 1987; Williams, 1987) and the wider press (Rogers, 1987; Van Gelder, 1988). However, the promise of HyperCard and its scripting language, HyperTalk, have yet to be fully exploited. In particular, the usefulness of HyperCard for various types of psychological research has not been determined. Martin (1988) has suggested that HyperCard might be suitable for the development of computerized psychological tests. The impetus for the present study was the authors' interest in exploring the limits of HyperCard's ability to rapidly modify visual stimuli and its utility in the administration of a psychological test of visual spatial skills.

\section{DEVELOPMENT}

Four general strategies for the modification of visual stimuli were explored, to see which one was most appropriate for the development of a computerized blockdesign task: the switching of cards, the use of the tools at the paint level, the switching of icon IDs, and the writing of strings to a field. In the first case, modification of a screen display is accomplished by opening another card. If one wishes to change the whole display, a completely new card may be created. If one wishes to change only a portion of the display, the card must be copied and pasted; modifications are then made to the new card. In either case, the visual display changes when one card is closed and another is opened. The time required to do this depends on numerous factors, including the hardware in use, the complexity of a card's contents, and the nature of handlers for closeCard and openCard messages. As the complexity of cards or handlers increases, the time required to close and open cards may substantially in-

Correspondence may be addressed to Thomas A. Martin, Department of Psychology, Susquehanna University, Selinsgrove, PA 17870. crease. While this strategy may be useful for some applications, it was judged to be unacceptably slow for tasks in which time is critical. In addition, it was inappropriate to the requirements of the block-design task in other respects.

The second strategy for modifying visual stimuli, use of the tools at the paint level, is also a relatively slow process. The browse tool is in use at most times because it is the tool that generates system messages. During the switch from the browse tool to the paint tools, one encounters a brief delay. Therefore, this strategy was also judged to be unsuitable for modification of visual stimuli during tasks in which time is critical.

The third approach allows modification of an area of a card no more than 32 pixels square. A button may display any icon contained in the HyperCard or created with a resource editor such as ResEdit or Icon Factory (Goodman, 1988). One can change the icon of a button from a HyperTalk script with a single command. This yields performance that is substantially faster than the previous strategies, but it is limited by the dimensions of icon resources.

The final strategy entails modifying visual stimuli by writing a character or string to a field. HyperCard allows the use of any available font within a field object. By using a font editor, such as ResEdit or FONTastic Plus, it is possible to create a font that contains the elements of the visual display one wishes to create rather than the usual set of characters. The font is then installed as a resource in the stack that will use it. This strategy was judged to be faster, more flexible, and more appropriate to the requirements of the block-design task, and was therefore adopted in the development of the block-design stackware.

The essential requirements of a block-design task are the presentation of a pattern to be copied by the subject, an object that can be manipulated by the subject to create elements of the design, and a means by which assembly can be accomplished. Manipulation of the object includes the rotation and repositioning of it on the screen. Ideally, the speed of the software and ease of its use should be 


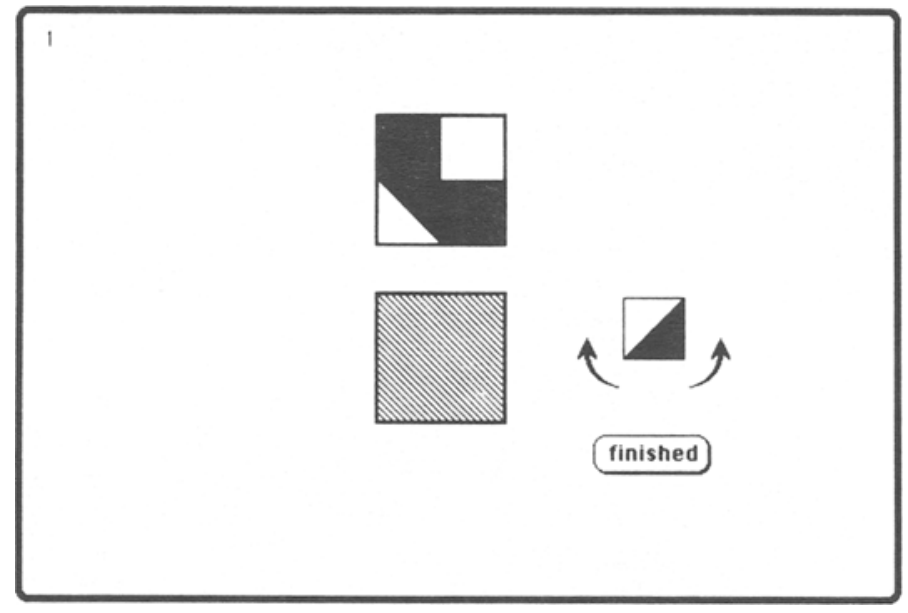

Figure 1. A four-block design.

sufficient to allow on-screen manipulation to occur as rapidly and easily as the handling of the actual physical object itself.

The block-design stack comprises 12 cards. The first card prompts entry of identifying information. The second allows the examiner to demonstrate the use of the mouse to rotate a block on the screen of the computer. The next 5 cards present four-block patterns, followed by three that present nine-block patterns (see Figures 1 and 2). In each case, the pattern to be copied is presented at the top of the screen, and the area in which the pattern is to be constructed is located immediately below it. To the right of the area in which the pattern is to be assembled is a single block. The mouse is used to effect twodimensional rotation of the block by clicking on the arrows located below it; when an arrow is clicked, the block rotates in the indicated direction. The block can then be copied into the pattern being assembled by means of clicking on the area of the screen in which it is to be located. Following the stimlus cards is one with a message thank- ing subjects for their participation in the research. The final card displays the subject's data. Three buttons on this card allow the experimenter to print the data, prepare to test another subject, or shut the system down. When the latter two buttons are activated, they display a dialogue box that asks whether the subject's data should be stored on disk.

After the completion of stackware development, its reliability and validity as a measure of visual spatial abilities was evaluated. It should not be assumed that the twodimensional rotation and pattern assembly permitted by this stackware adequately replicates an established threedimensional task.

\section{METHOD}

\section{Subjects}

Forty-seven college undergraduates were recruited from two introductory psychology classes at a small liberal arts university. Their incentive for participation was extra

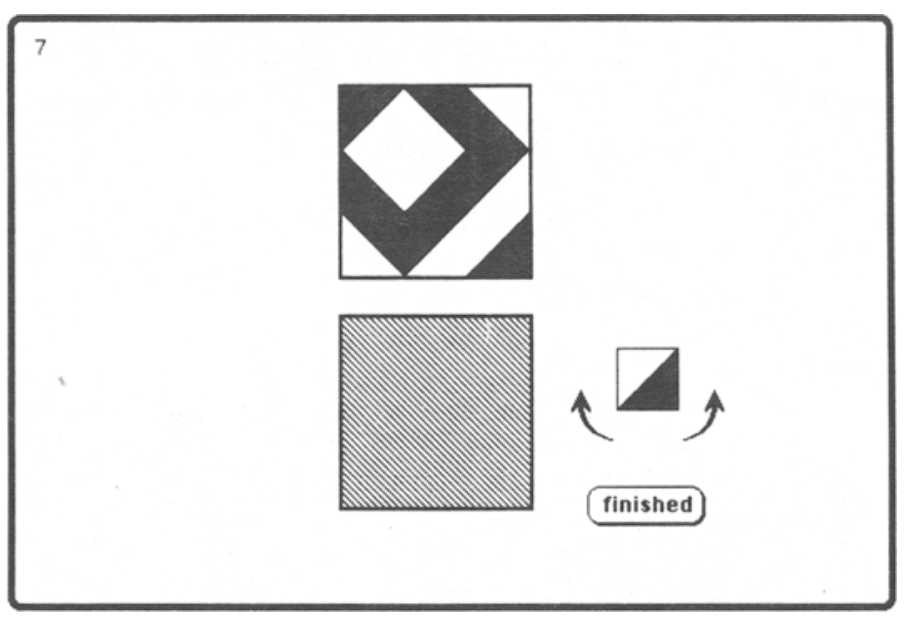

Figure 2. A nine-block design. 
credit offered by their instructors. Most who participated were first-semester freshmen. Three subjects' data were discarded; parts of two subjects' data were irretrievably lost to a scripting error, and a third subject was unable to complete the computerized block-design task properly, due to errors by an examiner. Of the 44 subjects whose data contributed the results reported below, 36 were female and 8 were male.

\section{Apparatus}

Two sets of apparatus were employed. The Wechsler Adult Intelligence Scale-Revised (WAIS-R) block-design subtest was administered with the standard materials provided in a WAIS-R kit.These include a small stimulus booklet and nine plastic cubes with which designs are to be assembled. The apparatus used to administer the computerized block-design task was an Apple Macintosh SE computer with two $800 \mathrm{~K}$ internal disk drives, $1 \mathrm{MB}$ RAM, a standard keyboard, and a standard Apple mouse. The mouse rested on a Mousetrak Mousepad (28.5 $\times$ $24 \mathrm{~cm})$.

The stackware with which the computerized blockdesign task was administered was scripted in version 1.0.1 of HyperTalk, the HyperCard scripting language (Goodman, 1988; Shafer, 1988). Since HyperCard immediately writes every change on a card to disk, and since accessing a floppy disk may momentarily freeze the cursor, version 1.1 of RAMstart was used to create a 100K RAM drive in which the block-design stack resided during the testing of subjects.

\section{Procedure}

The subjects were tested by one of three undergraduate examiners trained in the administration of both tasks. Each subject completed the block-design subtest of the WAIS-R and the block-design stackware. The order of presentation of the tasks was counterbalanced. Both tasks were presented during the same testing session, in the same laboratory; the typical testing session required 20 to $25 \mathrm{~min}$. The first eight designs of the WAIS-R subtest were administered in the standard manner (Wechsler, 1981). Construction of each design was timed with a stopwatch and recorded on a data sheet developed for this purpose.

At the outset of the computerized portion of the testing session, identifying information was entered by the examiner from the keyboard. The keyboard was then set aside, and the mousepad and mouse were placed before the comptuer. Each subject was seated directly in front of the computer in such a way that the mouse could be comfortably manipulated. The subjects were instructed in the use of the mouse and allowed to experiment with it. They were then shown how the block could be rotated with the mouse, and again they were given an opportunity to practice.

Instructions in the assembly of the computerized block designs closely followed those of the WAIS-R subtest. The first four-block design (see Figure 1) was displayed, and the examiner demonstrated the construction of the pattern. The newly assembled pattern was then erased, and the subject was asked to reassemble it and to click the "finished" button when he or she was done. When the subject clicked the "finished" button, the elapsed time and strings representing the stimulus and assembled designs were stored on the data card. The next design was then presented. As was the case with the WAIS-R subtest, the set of designs administered was composed of five four-block designs and three nine-block designs. Also in keeping with the WAIS-R, the four-block designs had time limits of $60 \mathrm{sec}$ and the nine-block designs had limits of $120 \mathrm{sec}$. The task was terminated after three consecutive failures or after all eight designs had been administered.

\section{RESULTS}

Two measures were calculated on the basis of the subjects' performance on each task: mean elapsed time for the assembly of designs and total number of correctly completed designs. Designs that were not attempted because the criterion of three consecutive failures had been met were assigned a value equal to the time limit for that design. Split-half reliability coefficients were calculated for each condition of administration and adjusted with the Spearman-Brown formula (see Table 1). They indicate that the stackware's reliability compares favorably to that of the WAIS-R subtest. Validity coefficients were derived by correlating measures from each form of administration. The validity coefficient for mean elapsed time was moderately strong ( $r=.68, p<.001)$, but that of correctly completed designs failed to reach significance $(r=.24)$.

A post hoc review of the data was conducted to identify possible explanations for the failure to achieve a significant validity coefficient for correctly completed designs. It was concluded that the low correlation may be attributable to two factors: restriction of range and commission of different types of errors during the two tasks. The mean number of correctly completed designs for each task was quite high and the variation was low (see Table 2). Under these conditions, high correlation coefficients would not be expected. Further review found that

Table 1

Split-Half Reliability Estimates of Block-Design Tasks

\begin{tabular}{lcc} 
& \multicolumn{2}{c}{ Measure } \\
\cline { 2 - 3 } Condition & Elapsed Time & Correct Completions \\
\hline Stackware task & .85 & .84 \\
WAIS-R subtest & .78 & .58 \\
\hline
\end{tabular}

Table 2

Number of Correctly Completed Designs

\begin{tabular}{lcc}
\hline \multicolumn{1}{c}{ Condition } & Mean & Standard deviation \\
\hline Stackware task & 6.49 & 1.46 \\
WAIS-R subtest & 7.11 & 0.97 \\
\hline
\end{tabular}

Note-The maximum score attainable was 8 . 
of 66 errors committed during the stackware task, all but one $(98 \%)$ were due to failure to complete the design within the established time limit. Of the 39 errors on WAIS-R designs, 25 errors (64\%) were the result of running out of time. Many of the remaining 14 errors (36\%) represent situations in which subjects reported that they had finished the design, but then noticed that they had misplaced a block. According to the directions for the WAIS$\mathrm{R}$ subtest, such a response must be scored as a failure. For some reason, the subjects were less prone to make this type of error during the stackware task. Consequently, it is possible that the measure of correct completions loads on somewhat different factors in the two tasks.

Finally, it must be noted that mean elapsed time differed substantially between the conditions. The mean elapsed time for assembly of the stackware designs was $58 \mathrm{sec}$, with a standard deviation of $10.4 \mathrm{sec}$. The WAIS-R designs were completed more rapidly, resulting in a mean elapsed time of $29 \mathrm{sec}$ and a standard deviation of $9.9 \mathrm{sec}$.

\section{DISCUSSION}

At the outset of this study, it was anticipated that the stackware administration of the block-design task would achieve reliability similar to that of the WAIS-R subtest, and that the validity coefficients would establish a moderate relationship between the measures. With the exception of the validity coefficient for correctly completed designs, these expectations were fulfilled, and the
HyperCard stackware functioned effectively as a tool for the measurement of visual spatial performance. Although HyperCard is likely not the development environment of choice for presentation of visual stimuli in tasks in which time is highly critical (e.g., tachistoscopic simulation), it easily handled the demands of the task that we studied. Our results suggest that HyperCard may be an appropriate environment for the development of computeradministered psychological tests, including those with a performance component.

\section{REFERENCES}

Atkinson, B. (1987). HyperCard [Computer program]. Cupertino, CA: Apple Computer.

Goodman, D. (1987, October). The two faces of HyperCard. Macworld, pp. 123-129.

Goodman, D. (1988). Danny Goodman's HyperCard developer's guide. New York: Bantam.

MarTIN, T. A. (1988, February). Scripting in HyperTalk: Applications in psychology. Paper presented at the Fourth Annual Psychology Conference of the University of Scranton, Scranton, PA.

Rogers,M. (1987, August 31). Hyper-excitement at Apple. Newsweek, p. 45 .

SAFFo, P. (1987, December). What you need to know about hypertext. Personal Computing, pp. 166-173.

ShAFER, D. (1988). HyperTalk programming. Indianapolis: Hayden. VAN Gelder, L. (1988, April). A feast of knowledge. Ms., p. 92.

Wechsler, D. (1981). WAIS-R manual. San Antonio, TX: Psychological Corporation.

Williams, G. (1987, December). HyperCard. Byte, pp. 109-117. 